\title{
AEROMAGNETIC MAP OF THE SOUTHINGTON QUADRANGLE, NEW HAVEN AND HARTFORD COUNTIES, CONNECTICUT
}

\author{
GEOPHYSICAL INVESTIGATIONS \\ MAP GP-854
}

